\title{
Modeling and Analysis of Ergodic Capacity in Network MIMO Systems
}

\author{
Kianoush Hosseini, Wei Yu, and Raviraj S. Adve \\ The Edward S. Rogers Sr. Department of Electrical and Computer Engineering \\ University of Toronto \\ Email: $\{$ kianoush, weiyu, rsadve $\} @$ comm.utoronto.ca
}

\begin{abstract}
This paper studies the downlink ergodic capacity of a network multiple-input multiple-output (MIMO) system. The system model includes base-stations (BSs) randomly distributed with a fixed density, each equipped with $M$ antennas, scheduling $K$ single-antenna users, and forming cooperating clusters via perfect backhaul links. Intra-cluster interference is eliminated by joint transmission using zero-forcing beamforming assuming perfect channel state information (CSI), while inter-cluster interference remains. This paper shows that although coordinating a large cluster of BSs eliminates strong interferers, the coordination gain depends crucially on the loading factor, defined as the ratio of $M$ and $K$. In particular, we show that with $M=K$, increasing the coordination cluster size is only beneficial for the cluster-edge users, while degrading the ergodic capacity of the users located close to the cluster center. In contrast, when $M>K$, increasing the cluster size potentially improves every user's ergodic capacity. In the second part of this paper, we use tools from stochastic geometry to completely characterize the user ergodic capacity in network MIMO systems. In this setting, we model the BS locations according to a homogeneous Poisson point process with a fixed density, and propose tractable, yet accurate, distribution functions for the signal and inter-cluster interference powers. We then derive an efficiently computable expression for the user ergodic capacity as a function of the distance between a user and the cluster center.
\end{abstract}

\section{INTRODUCTION}

Network multiple-input multiple-output (MIMO) is often hailed as a promising architecture to mitigate multicell interference and to bring significant improvements to future wireless cellular networks with dense deployment of basestations (BSs) [1]. By coordinating multiple BSs over the backhaul links, a network MIMO system can spatially distinguish multiple users across the BSs, thereby completely eliminate multicell interference. In this paper, we study the downlink of a network MIMO system where BSs form cooperating clusters. The main objective of this paper is to accomplish the following two tasks: (1) investigating the performance gain of the joint transmission scheme in a network MIMO system as a function of the given loading factor defined as the ratio of the transmit and receive antennas within each cluster; (2) deriving efficiently computable user ergodic capacity expression using tools from stochastic geometry.

The system model comprises a wireless cellular network wherein BSs are randomly deployed with a fixed density, and clustered using a regular lattice. Each BS is equipped with $M$

This work is supported in part by TELUS Canada and in part by Natural Science and Engineering Research Council (NSERC) of Canada. antennas, and schedules $K$ single-antenna users with $M \geq$ $K$. The scheduled users within a cluster are jointly served in the downlink via zero-forcing $(\mathrm{ZF})$ beamforming under perfect channel state information (CSI); users are subject to intercluster interference.

In the absence of CSI acquisition overhead and backhaul capacity/delay constraints, it is widely acknowledged that increasing the coordination cluster size introduces significant gain in terms of user ergodic capacity by eliminating the strong interferers. This paper shows, however, that this gain depends crucially on the ratio of $M$ and $K$. In particular, we distinguish between two distinct regimes: a fully-loaded system where $M=K$, and a system with an excess number of antennas equipped at each BS, i.e., $M>K$.

In a fully-loaded system, establishing joint transmission across a larger set of BSs brings two competing consequences. First, the inter-cluster interference power at each user location decreases. Second, as this paper shows, joint processing using $\mathrm{ZF}$ beamforming also introduces a penalty in terms of the signal power. Surprisingly, even without accounting for CSI acquisition overhead and backhaul impairment, the results of this paper show that as the cluster size goes to infinity, the ergodic capacity of every user in fact goes to zero asymptotically in a fully-loaded network MIMO system. In contrast, it converges to a non-zero constant when $M>K$. Our results imply that using an excess number of antennas at each BS is essential to realizing the cooperation gain of the network MIMO systems in future wireless networks.

This paper also aims to develop accurate, yet tractable, mathematical models for network MIMO systems that account for random locations of the BSs [2]. Although stochastic geometry has been accepted as a core mathematical tool to model such randomness in wireless networks [3], and has been extensively employed to investigate BS cooperation using interference coordination [4], non-coherent joint transmission [2], and distributed antenna systems [5], the performance of network MIMO systems has only been studied either using simplified Wyner models [6] or with extensive system-level simulations [7]. The main challenge in modeling network MIMO systems using stochastic geometry is the lack of tractable signal and interference distribution functions.

To address this issue, the second part of this paper considers a network MIMO system as described above wherein the BS locations are modeled according to a homogeneous Poisson point process (PPP) with a fixed density. We first develop 


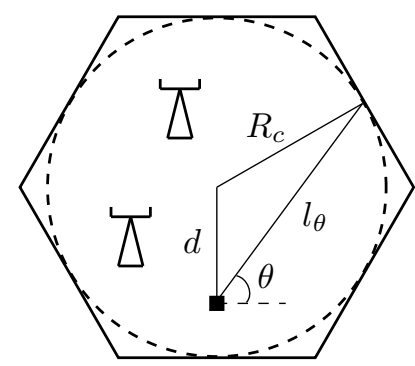

Fig. 1. A typical user is shown by the square marker and is located at distance $d$ from the center of a typical hexagonal cluster approximated by its inscribed circle of radius $R_{c}$. The rest of the $\mathbb{R}^{2}$ plane is the interference region.

approximate signal and inter-cluster interference distribution functions which, unlike the ones presented in [8], [9], are more amenable to analysis using stochastic geometry. We then derive an efficiently computable downlink user ergodic capacity expression in a network MIMO system under the two described regimes as a function of the user distance to the cluster center.

\section{SySTEM MODEL}

We consider the downlink of a cooperating cellular network wherein BSs are spatially distributed according to a homogeneous point process with a fixed density $\lambda$ over the entire $\mathbb{R}^{2}$ plane, where cooperating BS clusters are defined according to a regular hexagonal lattice. ${ }^{1}$ Each $\mathrm{BS}$ is equipped with $M$ transmit antennas, schedules $K$ single-antenna users, and has constrained power $P_{T}{ }^{2}$ The BSs within each cluster form a network MIMO system wherein intra-cluster interference cancellation is enabled by joint data transmission to the scheduled users using ZF beamforming. This paper assumes that perfect CSI is available at the BSs without incurring any overhead, and the BSs are connected using infinite-capacity and delay-free backhaul links. Further, clusters are subject to a sum-power constraint and allocate their powers equally across the downlink beams. In this paper, we focus on the ergodic capacity of a typical user $i$ located at distance $d$ from the center of cluster $l$. In addition, for analysis, the typical cluster is approximated by its inscribed circle of radius $R_{c}$. A snapshot of a typical cluster is depicted in Fig. 1.

The channel vector between BS $m$ in cluster $j$ to user $i$ in cluster $l$ is defined as $\mathbf{g}_{i l m j}=\sqrt{\beta_{i l m j}} \mathbf{h}_{i l m j} \in \mathcal{C}^{M}$ where $\mathbf{h}_{i l m j}$ denotes the small-scale Rayleigh channel fading, and consists of independent and identically distributed (i.i.d.) $\mathcal{C N}(0,1)$ components, $\beta_{i l m j}=\left(1+r_{i l m j} / D\right)^{-\alpha}$ denotes the path-loss coefficient with $D=1$ indicating the reference distance, $r_{i l m j}$ is the distance between BS $m$ in cluster $j$ and the typical user, and $\alpha>2$ is the path-loss exponent. For exposition, we assume that cluster $l$ comprises $B_{l}$ cooperating BSs. The composite channel matrix of cluster $l$ is denoted by $\mathbf{G}_{l}=\left[\mathbf{g}_{1 l}, \ldots, \mathbf{g}_{K_{l} l}\right] \in \mathcal{C}^{B_{l}(M \times K)}$, where

\footnotetext{
${ }^{1}$ Any clustering approach that partitions the network coverage area can be used.

${ }^{2}$ We assume that the user density is sufficiently large such that each BS schedules $K$ users from within its cluster area.
}

$K_{l}=K B_{l}$, and $\mathbf{g}_{i l}=\left[\mathbf{g}_{i l 1 l}^{\top}, \ldots, \mathbf{g}_{i l B_{l} l}^{\top}\right]^{\top} \in \mathcal{C}^{M B_{l}}$ indicates the collective channel vector between the $B_{l}$ serving BSs and user $i$. The downlink ZF beams are designed as the scaled pseudo-inverse of the channel matrix; $\mathbf{w}_{i l}$ denotes the unit-norm beam assigned for user $i$ in cluster $l$. We further define $\mathbf{f}_{i l j}=\left[\mathbf{g}_{i l 1 j}^{\top}, \ldots, \mathbf{g}_{i l B_{j} j}^{\top}\right]^{\top}$ as the collective interference channel vector from the $B_{j}$ BSs in cluster $j$ to the typical user. The SINR of the typical user is therefore given by

$$
\gamma_{i l}=\frac{\rho\left|\mathbf{g}_{i l}^{\mathrm{H}} \mathbf{w}_{i l}\right|^{2}}{\sum_{j \in \mathcal{C}_{l}} \sum_{k=1}^{K B_{j}} \rho\left|\mathbf{f}_{i l j}^{\mathrm{H}} \mathbf{w}_{k j}\right|^{2}+1}
$$

where $\mathcal{C}_{l}$ indicates the set of clusters interfering with the users in cluster $l, \rho=\frac{P_{T}}{K \sigma^{2}}$ denotes the signal-to-noise ratio, and $\sigma^{2}$ is the noise power. Finally, invoking the orthogonality principle of ZF beamforming, i.e., $\mathbf{w}_{i l} \perp$ span $\left\{\mathbf{g}_{k l}\right\}_{k \neq i}$, the dimension of the beamforming space for each user in cluster $l$ is $\zeta_{l}=$ $B_{l}(M-K)+1$.

To analyze the coordination gain of a network MIMO system, obtaining the distribution functions of both signal power $\left|\mathbf{g}_{i l}^{\mathrm{H}} \mathbf{w}_{i l}\right|^{2}$ and interference power produced by individual beam transmissions in interfering clusters $\left|\mathbf{f}_{i l j}^{\mathrm{H}} \mathbf{w}_{k j}\right|^{2}$ is essential. In the following section, we revisit the approach proposed in [8] to obtain approximate signal and interference distributions in a network MIMO system under a fixed BS deployment.

\section{Signal And Interference Power Distributions: A FIXED BS DEPLOYMENT}

The distributions of the signal and interference powers in a wireless network where channel vectors are isotropic, i.e., only consist of i.i.d. components, have been derived previously (refer to [10, Proof of Theorem 1] for more details). The importance of the isotropic assumption is twofold. First, obtaining the distribution of the channel strength becomes straightforward. Second, deriving the exact distribution of the power of the channel vector projected onto a $\mathrm{ZF}$ beamforming space becomes tractable.

In a network MIMO system, however, each composite channel vector is subject to different path-loss components, i.e., the channel vectors are non-isotropic. As a consequence, characterizing the exact signal and interference power distributions is much more difficult. In particular, for a fixed realization of a cellular network as presented in the preceding section, the intended channel strength $\left\|\mathbf{g}_{i l}\right\|^{2}=\sum_{b}\left\|\mathbf{g}_{i l b l}\right\|^{2}$ and each interference channel strength $\left\|\mathbf{f}_{i l j}\right\|^{2}=\sum_{m}\left\|\mathbf{g}_{i l m j}\right\|^{2}$ are, respectively, the sum of $B_{l}$ and $B_{j}$ independent, but nonidentically distributed, Gamma random variables. Although the exact distribution of the sum of non-identically distributed Gamma random variables does not yield a mathematically tractable expression, a second-order moment matching approach proposed in [11] can be employed to obtain an efficient approximation as follows.

Lemma 1: Let $\left\{X_{i}\right\}_{i=1}^{m}$ be a set of $m$ independent random variables such that $X_{i} \sim \Gamma\left(k_{i}, \theta_{i}\right)$. Then, $Y=\sum_{i} X_{i}$ has the same first and second order statistics as a Gamma random variable with the shape and scale parameters given as

$$
k=\frac{\left(\sum_{i} k_{i} \theta_{i}\right)^{2}}{\sum_{i} k_{i} \theta_{i}^{2}} \text { and } \theta=\frac{\sum_{i} k_{i} \theta_{i}^{2}}{\sum_{i} k_{i} \theta_{i}} .
$$


Approximation 1: Using Lemma 1, the sum of $m$ independent but non-identically distributed Gamma random variables can be approximated as a Gamma random variable with the effective shape and scale parameters as presented in (2).

By adopting Approximation 1, it follows that $\left\|\mathbf{g}_{i l}\right\|^{2} \sim$ $\Gamma\left(k_{i l}, \theta_{i l}\right)$ and $\left\|\mathbf{f}_{i l j}\right\|^{2} \sim \Gamma\left(k_{i j}, \theta_{i j}\right)$ with

$$
\begin{aligned}
& k_{i l}=M \frac{\left(\sum_{b=1}^{B_{l}} \beta_{i l b l}\right)^{2}}{\sum_{b=1}^{B_{l}} \beta_{i l b l}^{2}}, \quad \theta_{i l}=\frac{\sum_{b=1}^{B_{l}} \beta_{i l b l}^{2}}{\sum_{b=1}^{B_{l}} \beta_{i l b l}} \\
& k_{i j}=M \frac{\left(\sum_{m=1}^{B_{j}} \beta_{i l m j}\right)^{2}}{\sum_{m=1}^{B_{j}} \beta_{i l m j}^{2}}, \quad \theta_{i j}=\frac{\sum_{m=1}^{B_{j}} \beta_{i l m j}^{2}}{\sum_{m=1}^{B_{j}} \beta_{i l m j}} .
\end{aligned}
$$

From (3) and (4), it can be shown that $k_{i l} \leq M B_{l}$ and $k_{i j} \leq M B_{j}$ with equality if the two vectors were isotropic. In essence, each spatial dimension (i.e., each component) of an isotropic vector contributes 1 to the shape parameter of the power distribution, while the contribution of each spatial dimension of a non-isotropic vector to the shape parameter is less than 1.

Based on the above observation and in order to further facilitate the characterization of power distributions in a network MIMO system, this paper adopts the following additional approximation pioneered in [8] which treats $\mathbf{g}_{i l}$ and $\mathbf{f}_{i l j}$ as isotropic vectors, while their shape parameters are modified to reflect the non-isotropic nature of the channels.

Approximation 2: The intended channel vector $\mathbf{g}_{i l}$ can be approximated as an isotropic vector distributed as $\mathcal{C N}\left(\mathbf{0}, \theta_{i l} \mathbf{I}_{M B_{l}}\right)$ where each spatial dimension adds $k_{i l} / M B_{l}$ to the shape parameter of the power distribution. Similarly, the interference channel $\mathbf{f}_{i l j}$ can be treated as an isotropic vector distributed as $\mathcal{C N}\left(\mathbf{0}, \theta_{i j} \mathbf{I}_{M B_{j}}\right)$ where each spatial dimension adds $k_{i j} / M B_{j}$ to the shape parameter of the power distribution.

By treating the channel vectors as if their components were i.i.d., and noting that the ZF beam designed for each user in cluster $l$ lies in a $B_{l}(M-K)+1$ dimensional subspace, whereas any interfering beam lies in a one-dimenstional subspace [10], the power distribution of the channel projected onto the ZF beam space can now be characterized as follows:

Lemma 2: Under Approximations 1 and 2, the signal power of the typical user and interference power produced by transmission of a single beam in cluster $j$ seen at the typical user are, respectively, distributed as

$$
\begin{aligned}
\left|\mathbf{g}_{i l}^{\mathrm{H}} \mathbf{w}_{i l}\right|^{2} & \sim \Gamma\left(\frac{k_{i l}\left(B_{l}(M-K)+1\right)}{M B_{l}}, \theta_{i l}\right) \\
\left|\mathbf{f}_{i l j}^{\mathrm{H}} \mathbf{w}_{k j}\right|^{2} & \sim \Gamma\left(\frac{k_{i j}}{M B_{j}}, \theta_{i j}\right) .
\end{aligned}
$$

The presented signal distribution function in (5) allows us to upper bound the ergodic capacity and investigate its limiting behavior as the cluster size increases under both $M=K$ and $M>K$ operating regimes.

\section{Two Operating Regimes: $M=K$ versus $M>K$}

It is well accepted that establishing coordination across a large number of BSs leads to suppression of strong intercluster interference. This section aims at understanding how this might affect the received signal power, and therefore the user ergodic capacity, at each user location. To address this question, we assume that $B_{l}=\lambda \pi R_{c}^{2}$ BSs are uniformly distributed within the typical cluster $l$. We first focus on user $i$ located at the cluster center. The following theorem presents the expected signal power $\left|\mathbf{g}_{i l}^{\mathrm{H}} \mathbf{w}_{i l}\right|^{2}$ for sufficiently large $R_{c}$.

Theorem 1: Under Approximations 1 and 2, and for sufficiently large $R_{c}$, we have that

$$
\begin{aligned}
& \mathbb{E}\left[\left|\mathbf{g}_{i l}^{\mathrm{H}} \mathbf{w}_{i l}\right|^{2}\right]= \\
& \quad \frac{2\left(\lambda \pi R_{c}^{2}(M-K)+1\right)}{R_{c}^{2}(\alpha-1)(\alpha-2)}\left[1-\frac{1+(\alpha-1) R_{c}}{\left(1+R_{c}\right)^{\alpha-1}}\right] .
\end{aligned}
$$

Proof: Using the signal power distribution function (5), we have that

$$
\begin{aligned}
\mathbb{E}\left[\left|\mathbf{g}_{i l}^{\mathrm{H}} \mathbf{w}_{i l}\right|^{2}\right] & \stackrel{(a)}{=} \frac{B_{l}(M-K)+1}{B_{l}} \sum_{b=1}^{B_{l}}\left(1+r_{i l b l}\right)^{-\alpha} \\
& \stackrel{(b)}{=}\left(B_{l}(M-K)+1\right) \mathbb{E}\left[(1+r)^{-\alpha}\right] \\
& \stackrel{(c)}{=} \frac{2\left(\lambda \pi R_{c}^{2}(M-K)+1\right)}{R_{c}^{2}} \int_{0}^{R_{c}}(1+r)^{-\alpha} r \mathrm{~d} r .
\end{aligned}
$$

Here, (a) follows from noting that if $X \sim \Gamma(k, \theta)$, then $\mathbb{E}(X)=k \theta$. Relation $(b)$ follows from the law of large numbers. In $(c)$, we use the fact that with $B_{l}$ uniformly distributed BSs, the distance probability distribution function for a user at the center is given by $f_{r}(r)=2 r / R_{c}^{2}$. Finally, noting that

$$
\int \frac{x}{(1+x)^{a}} \mathrm{~d} x=\frac{(1+x)^{1-a}(-a x+x-1)}{(a-1)(a-2)}
$$

the proof is complete.

Remark 1: For sufficiently large $R_{c}$, Theorem 1 is essentially valid for all users located close to the cluster center. The distribution of the BS locations, however, is not symmetric around the cluster-edge users. Therefore, slightly increasing the cluster size provides them with an opportunity to gain from more serving BSs, thereby enhancing the ergodic capacity. However, by further increasing the cluster size, the clusteredge users eventually become cluster-center users. Hence, Theorem 1 eventually applies.

Remark 2: Based on Theorem 1, with $M=K$, it can be seen that the expected signal power is a decreasing function in $R_{c}$. As a result, in a fully-loaded system, increasing the cluster size introduces a penalty in terms of the received signal power.

To elaborate on this result, it should be noted that the signal power of a typical user $\left|\mathbf{g}_{i l}^{\mathrm{H}} \mathbf{w}_{i l}\right|^{2}$ depends on the intended channel strength and the dimension of the beamforming space. Therefore, investigating the connection between the cluster size and these two factors provides further insight. As the first step, the following theorem shows that the indented channel strength $\left\|\mathbf{g}_{i l}\right\|^{2}$ converges to a constant as the cluster size grows.

Theorem 2: Under Approximation 1, and for sufficiently large $R_{c}$, it follows that

$$
\mathbb{E}\left[\left\|\mathbf{g}_{i l}\right\|^{2}\right]=\frac{2 \pi \lambda M}{(\alpha-1)(\alpha-2)} .
$$


Proof:

$$
\begin{aligned}
\mathbb{E}\left[\left\|\mathbf{g}_{i l}\right\|^{2}\right] & \stackrel{(a)}{=} M \sum_{b=1}^{B_{l}}\left(1+r_{i l b l}\right)^{-\alpha} \stackrel{(b)}{=} M B_{l} \mathbb{E}\left[(1+r)^{-\alpha}\right] \\
& \stackrel{(c)}{=} 2 \pi \lambda M \int_{0}^{R_{c}}(1+r)^{-\alpha} r \mathrm{~d} r \\
& \stackrel{(d)}{=} \frac{2 \pi \lambda M}{(\alpha-1)(\alpha-2)}\left[1-\frac{1+R_{c}(\alpha-1)}{\left(1+R_{c}\right)^{\alpha-1}}\right]
\end{aligned}
$$

where $(a)$ is based on the expected value of a Gamma distribution presented in (3), and $(b)$ is based on the law of large numbers. Noting that $B_{l}$ BSs are scattered uniformly within a cluster, $(c)$ follows using the distance distribution. Using the integral expression in (7), $(d)$ holds. Finally, assuming sufficiently large $R_{c}$, the second term can be ignored, which completes the proof.

Remark 3: Theorem 2 states that, while the dimension of the channel vector increases as more BSs participate in serving a user, the channel norm becomes a constant. In other words, the spatial dimensions provided by the BSs located far away from a user do not contribute to the channel strength.

The second important factor is the dimension of the beamforming space $\zeta_{l}$ for each user (Recall that $\zeta_{l}=B_{l}(M-K)+$ $1)$. When $M=K, \zeta_{l}=1$; the intended channel vector and the $\mathrm{ZF}$ beam assigned to a user are therefore independent.

As a consequence, as $R_{c}$ grows, the signal power $\left|\mathbf{g}_{i l}^{\mathrm{H}} \mathbf{w}_{i l}\right|^{2}$ is the power of the inner product of two independent and constant-norm vectors in spaces of increasing dimension; hence, the signal power degrades.

Theorem 1 can be adopted to examine the asymptotic ergodic capacity of a typical user at a finite distance $d$ from the cluster center $\mathcal{C}_{i}(d)$ in a fully-loaded network MIMO system as follows:

Corollary 1: Under Approximations 1 and 2, the asymptotic ergodic capacity of a user located $d$ meters away from the cluster center in a fully-loaded network MIMO system with $M=K$ goes to zero as the cluster size goes to infinity.

Proof: We have

$$
\begin{gathered}
\mathcal{C}_{i}(d)=\mathbb{E}\left[\log \left(1+\gamma_{i l}\right)\right] \stackrel{(a)}{\leq} \mathbb{E}\left[\log \left(1+\rho\left|\mathbf{g}_{i l}^{\mathrm{H}} \mathbf{w}_{i l}\right|^{2}\right)\right] \\
\stackrel{(b)}{\leq} \log \left(1+\rho \mathbb{E}\left[\left|\mathbf{g}_{i l}^{\mathrm{H}} \mathbf{w}_{i l}\right|^{2}\right]\right) \\
\stackrel{(c)}{=} \log \left(1+\frac{2 \rho}{R_{c}^{2}(\alpha-1)(\alpha-2)}\left[1-\frac{1+(\alpha-1) R_{c}}{\left(1+R_{c}\right)^{\alpha-1}}\right]\right)
\end{gathered}
$$

where $(a)$ is obtained by ignoring the inter-cluster interference, $(b)$ is obtained by the Jensen's inequality, and $(c)$ follows from Theorem 1 with $M=K$. The final expression converges to zero as $R_{c}$ increases. Thus, $\lim _{R_{c} \rightarrow \infty} \mathcal{C}_{i}(d)=0$.

Our result illustrates that by increasing the cluster size, the ergodic capacity of every user reduces in a fully-loaded network MIMO system. In particular, the signal power penalty eventually outweighs the gain in reducing interference power as the cluster size increases.

In contrast, from Theorem 1 , for sufficiently large $R_{c}$, the expected signal power converges to a constant when $M>K$ as

$$
\mathbb{E}\left(\left|\mathbf{g}_{i l}^{\mathrm{H}} \mathbf{w}_{i l}\right|^{2}\right)=\frac{2 \pi \lambda(M-K)}{(\alpha-1)(\alpha-2)} .
$$

The reason is that while the channel strength becomes a constant as $R_{c}$ increases, $\zeta_{l}$ grows at the same rate as the number of coordinating BSs. Therefore, the unit-norm ZF beam lies in a subspace whose dimension becomes larger as $R_{c}$ increases. As a consequence, when excess antennas are available at the BSs, increasing the cluster size, up to the point where the signal power becomes a constant and interference power reaches the noise level, improves the ergodic capacity of each user. The user ergodic capacity essentially becomes a constant beyond this point.

\section{USER ERGodic CAPACITY: A Stochastic GeOMETRY APPROACH}

The previous section investigated the properties of the users ergodic capacity in two distinct operating regimes in a network MIMO system. This section of the paper develops an efficiently computable formulae for a user's ergodic capacity (averaged over both BS locations and fading) using tools from stochastic geometry. We assume that the BSs are distributed according to a homogeneous PPP $\Phi$ with density $\lambda$. Since the parameters of the approximate distributions presented in (5) and (6) are functions of the joint distances between the typical user and a set of cooperating BSs, a tractable characterization of ergodic capacity using these distributions is not feasible. To tackle this issue, we first propose alternative signal and interference power distributions.

\section{A. Proposed Signal Power Distribution}

Let us consider a fixed realization of the PPP $\Phi$ with $B_{l}$ BSs in a typical cluster. The following proposition introduces an alternative signal power distribution function in a network MIMO system.

Proposition 1: Using Approximation 1, $\left|\mathbf{g}_{i l}^{\mathrm{H}} \mathbf{w}_{i l}\right|^{2} \stackrel{d}{=}$ $\sum_{b=1}^{B_{l}} \beta_{i l b l} S_{b}{ }^{3}$, where

$$
S_{b} \sim \Gamma\left(\frac{B_{l}(M-K)+1}{B_{l}}, 1\right) .
$$

Proof: Applying Approximation 1, it can be shown that $\sum_{b=1}^{B_{l}} \beta_{i l b l} S_{b}$ is distributed as the Gamma random variable in (5).

The distribution of each term in $\sum_{b=1}^{B_{l}} \beta_{i l b l} S_{b}$ is now a function of the distance between the typical user and only one of the serving BSs. However, $B_{l}$ is a (Poisson) random variable. Hence, the signal power distribution is still a function of individual realizations of $\Phi$, making further analysis intractable. To address this issue, we further replace $B_{l}$ by its expected value.

Approximation 3: We approximate $\quad\left|\mathbf{g}_{i l}^{\mathrm{H}} \mathbf{w}_{i l}\right|^{2}$ as $\sum_{b=1}^{B_{l}} \beta_{i l b l} \tilde{S}_{b}$, where

$$
\tilde{S}_{b} \sim \Gamma\left(\frac{\bar{B}_{l}(M-K)+1}{\bar{B}_{l}}, 1\right)
$$

\footnotetext{
${ }^{3}$ The equivalence in distribution is indicated by $\stackrel{d}{=}$.
} 
where $\bar{B}_{l}=\lambda \pi R_{c}^{2}$ denotes the expected number of BSs in cluster $l$.

The remainder of this section is devoted to obtaining an efficient interference power distribution function.

\section{B. Proposed Interference Power Distribution}

Since interference produced by different clusters are independent, we focus on the interference power from the $B_{j}$ cooperating BSs in cluster $j$.

First, note that the typical user receives $K B_{j}$ interfering beams from cluster $j$ during each time slot denoted as $I_{i l}^{j}=$ $\sum_{k=1}^{K B j}\left|\mathbf{f}_{i l j}^{\mathrm{H}} \mathbf{w}_{k j}\right|^{2}$. Since the $K B_{j} \mathrm{ZF}$ beams are not necessarily orthogonal, $I_{i l}^{j}$ is not, in general, a summation of independent terms. Obtaining its distribution therefore is not mathematically feasible. To obtain an approximate distribution, however, similar to the related works, e.g., [8]-[11], we approximate the interfering beams as orthogonal vectors. ${ }^{4}$

Approximation 4: We approximate $I_{i l}^{j}$ as the summation of $K B_{j}$ i.i.d. Gamma random variables, where the $k^{\text {th }}$ term is distributed as in (6).

Under Approximation 4, we therefore have

$$
I_{i l}^{j} \sim \Gamma\left(\frac{K}{M} k_{i j}, \theta_{i j}\right) .
$$

Again, since the parameters of the approximate interference power distribution in (10) are complex functions of distances between a user and the BSs in cluster $j$, it cannot be directly used to obtain the ergodic capacity expression through stochastic geometry approach. Hence, we propose to further approximate the interference power distribution as follows:

Proposition 2: Under Approximations 1 and 4, we have

$$
I_{i l}^{j} \stackrel{d}{=} \sum_{m=1}^{B_{j}} \beta_{i l m j} I_{i l m j}
$$

where $I_{i l m j} \sim \Gamma(K, 1)$.

Proof: Using Approximation 1, it can be shown that $\sum_{m=1}^{B_{j}} \beta_{i l m j} I_{i l m j}$ is distributed as the Gamma random variable in (10).

Remark 4: From Proposition 2, $I_{i l}^{j}$ is equal in distribution to the interference power produced by the same set of BSs as in cluster $j$ where each BS independently serves its $K$ scheduled users using $\mathrm{ZF}$ beamforming. We therefore have that

$$
\sum_{j \in \mathcal{C}_{l}} \sum_{m=1}^{B_{j}} \beta_{i l m j} I_{i l m j} \stackrel{d}{=} \sum_{m \in \Phi \backslash \Phi_{l}}\left(1+r_{i l m}\right)^{-\alpha} X_{i l m}
$$

where $X_{i l m} \sim \Gamma(K, 1), \Phi_{l}$ is the set of cooperating BSs in cluster $l$, and $r_{i l m}$ denotes the distance between the interfering BS $m$ and the typical user.

Based on the proposed approximate signal and interference power distributions, the next section characterizes the ergodic capacity expression in a network MIMO system.

\footnotetext{
${ }^{4}$ In fact, scheduling a set of semi-orthogonal users enhances the performance of a network under ZF beamforming [12].
}

\section{User Ergodic Capacity Expression}

We now derive the ergodic capacity expression for a user located at distance $d$ from the cluster center. It is notable that this expression would allow a theoretically rigorous way of investigating the achievable ergodic capacity of a network MIMO system at every location of a cluster.

Theorem 3: For the given system parameters $M, K, \lambda$, and $\alpha$, the ergodic capacity of a user located at the distance $d \leq R_{c}$ from the cluster center is given by

$$
\begin{aligned}
& \mathcal{C}_{i}(d)=\int_{0}^{\infty} \frac{e^{-z}}{z} \exp \left(-\lambda \int_{0}^{2 \pi} \int_{l(\theta)}^{\infty}\left[1-\psi_{K}(z, r)\right] r \mathrm{~d} r \mathrm{~d} \theta\right) \\
& \times\left\{1-\exp \left(-\lambda \int_{0}^{2 \pi} \int_{0}^{l(\theta)}\left[1-\psi_{\omega_{l}}(z, r)\right] r \mathrm{~d} r \mathrm{~d} \theta\right)\right\} \mathrm{d} z
\end{aligned}
$$

where $\psi_{n}(z, r)=\left(1+\rho z(1+r)^{-\alpha}\right)^{-n}, \omega_{l}$ indicates the shape parameter in (9), and $l(\theta)=\sqrt{R_{c}^{2}-d^{2} \cos ^{2} \theta}+d \sin \theta$ indicates the distance of the typical user from the cluster edge for a given angle $\theta$ as depicted in Fig. 1.

Proof: The proof is presented in Eq. (13) on the next page, where $(a)$ follows from using $\log (1+x)=$ $\int_{0}^{\infty} \frac{e^{-t}}{t}\left(1-e^{-x t}\right) \mathrm{d} t$ [13]. In $(b)$, we use the change of variable $t=z\left(\sum_{j \in \mathcal{C}_{l}} \sum_{k=1}^{K B_{j}} \rho\left|\mathbf{f}_{i l j}^{\mathrm{H}} \mathbf{w}_{k j}\right|^{2}+1\right)$. Further, since the integrand is non-negative, the order of integration and expectation can be exchanged. The expression in (13b) involves moment generating functions (MGF) of the signal and interference powers. Therefore, in $(c)$, we replace the signal and interference powers with their equivalent terms (in distribution) as, respectively presented in (9) and (11). Since $\Phi$ is independent of both $X_{i l m}$ and $\tilde{S}_{b}$, we have relation $(d)$. Noting that $\tilde{S}_{b}$ and $X_{i l m}$ are Gamma random variables, $(e)$ follows from the MGF of the Gamma distribution. Finally, using the probability generating functional of a homogenous PPP with density $\lambda$ [3] as given by

$$
\mathbb{E}\left[\prod_{x \in \Phi} v(x)\right]=\exp \left(-\lambda \int_{\mathbb{R}^{2}}[1-v(x)] \mathrm{d} x\right)
$$

and converting the coordinates from Cartesian to polar, we have the expression in (12).

\section{NUMERICAL VALIDATION}

We now present numerical results to validate the main conclusions and analytical results of this paper. In our numerical simulations, the number of cooperating BSs within each cluster is drawn according to a Poisson process with density $\lambda$. The average number of cooperating BSs ranges from 5 at cluster radius of 400 meters to 31 at cluster radius of 1000 meters. Each BS is equipped with $M$ antennas and schedules $K$ single antenna users. Joint downlink transmission takes place over the shared spectrum of bandwidth $W$ with frequency reuse factor of one. The ergodic capacity of a fixed typical user is averaged over different realizations of the Poisson process, BS locations, other users' locations, and channel fading. The analytical 


$$
\begin{aligned}
& \mathcal{C}_{i}(d) \stackrel{(a)}{=} \mathbb{E}_{\Phi, \mathbf{g}}\left[\int_{0}^{\infty} \frac{e^{-t}}{t}\left(1-\exp \left(-t \frac{\rho\left|\mathbf{g}_{i l}^{\mathrm{H}} \mathbf{w}_{i l}\right|^{2}}{\sum_{j \in \mathcal{C}_{l}} \sum_{k=1}^{K B_{j}} \rho\left|\mathbf{f}_{i l j}^{\mathrm{H}} \mathbf{w}_{k j}\right|^{2}+1}\right)\right) \mathrm{d} t\right] \\
& \stackrel{(b)}{=} \int_{0}^{\infty} \frac{e^{-z}}{z} \underbrace{\mathbb{E}_{\Phi, \mathbf{g}}\left[\exp \left(-z \rho \sum_{j \in \mathcal{C}_{l}} \sum_{k=1}^{K B_{j}}\left|\mathbf{f}_{i l j}^{\mathrm{H}} \mathbf{w}_{k j}\right|^{2}\right)\right]}_{\text {MGF of aggregate interference power }}[1-\underbrace{\mathbb{E}_{\Phi, \mathbf{g}}\left[\exp \left(-z \rho\left|\mathbf{g}_{i l}^{\mathrm{H}} \mathbf{w}_{i l}\right|^{2}\right)\right]}_{\text {MGF of the signal power }}] \mathrm{d} z \\
& \stackrel{(c)}{=} \int_{0}^{\infty} \frac{e^{-z}}{z} \mathbb{E}_{\Phi, X_{i l m}}\left[\exp \left(-z \rho \sum_{m \in \Phi \backslash \Phi_{l}}\left(1+r_{i l m}\right)^{-\alpha} X_{i l m}\right)\right]\left[1-\mathbb{E}_{\Phi, \tilde{S}_{b}}\left[\exp \left(-z \rho \sum_{b \in \Phi_{l}}\left(1+r_{i l b l}\right)^{-\alpha} \tilde{S}_{b}\right)\right]\right] \mathrm{d} z \quad(13 \mathrm{c}) \\
& \stackrel{(d)}{=} \int_{0}^{\infty} \frac{e^{-z}}{z} \mathbb{E}_{\Phi}\left[\prod_{m \in \Phi \backslash \Phi_{l}} \mathbb{E}_{X_{i l m}}\left[\exp \left(-z \rho\left(1+r_{i l m}\right)^{-\alpha} X_{i l m}\right)\right]\right]\left[1-\mathbb{E}_{\Phi}\left[\prod_{b \in \Phi_{l}} \mathbb{E}_{\tilde{S}_{b}}\left[\exp \left(-z \rho\left(1+r_{i l b l}\right)^{-\alpha} \tilde{S}_{b}\right)\right]\right]\right] \mathrm{d} z \\
& \stackrel{(e)}{=} \int_{0}^{\infty} \frac{e^{-z}}{z} \mathbb{E}_{\Phi}\left[\prod_{m \in \Phi \backslash \Phi_{l}}\left(1+z \rho\left(1+r_{i l m}\right)^{-\alpha}\right)^{-K}\right]\left[1-\mathbb{E}_{\Phi}\left[\prod_{b \in \Phi_{l}}\left(1+z \rho\left(1+r_{i l b l}\right)^{-\alpha}\right)^{-\omega_{l}}\right]\right] \mathrm{d} z .
\end{aligned}
$$

results are derived using (12). The system parameters are summarized in Table I.

Single Cluster Network: First, we consider a single circular cluster, i.e., inter-cluster interference is ignored. For this example, the user is located at the cluster center. Fig. 2 shows the ergodic capacity of this user as a function of cluster radius. As can be seen from the figure, the user ergodic capacity improves, then saturates as $R_{c}$ grows in a network MIMO system with excess number of antennas at each BS. However, when $M=K$, increasing the cluster size reduces the ergodic capacity. Fig. 2 also presents the analytical results. Note that in using expression (12), the interference power is set to zero to ignore inter-cluster interference. As shown in the figure, the analytical and numerical results perfectly match when $M>K$. With $M=K$, although there is a gap, both numerical and analytical results illustrate that ergodic capacity degrades as cluster size increases. This is due to the penalty in terms of the signal power imposed by increasing the cluster size.

Multi-Cluster Network: Next, we consider a network MIMO system comprising 19 hexagonal clusters, and study the ergodic capacity of a typical user located, respectively, at $d=0$ and $d=350$ meters away from the center (a cluster-edge user) of the typical cluster as a function of $R_{c}$.

As shown in Fig. 3, in a fully-loaded system, increasing $R_{c}$ to up to 600 meters improves the ergodic capacity of the cluster-edge user by reducing inter-cluster interference. However, with further increasing $R_{c}$, the penalty in signal power eventually outweighs the reduction in interference. Therefore, ergodic capacity eventually decreases. For the cluster-center user, reduction in interference by increasing the cluster radius is not sufficient to compensate for the reduction in the signal power. As a result, its ergodic capacity decreases as $R_{c}$ increases.

With $M>K$, the ergodic capacity is enhanced with increasing $R_{c}$. However, as seen from the figure, this gain is diminishing. The reason is that the improvement in terms
TABLE I

NETWORK DESIGN PARAMETERS

\begin{tabular}{|c|c|}
\hline BS density & $\lambda=10^{-5} \mathrm{~m}^{-2}$ \\
\hline Total bandwidth & $W=20 \mathrm{MHz}$ \\
\hline BS Max available power & $43 \mathrm{dBm}$ \\
\hline Background noise & $N_{o}=-174 \mathrm{dBm} / \mathrm{Hz}$ \\
\hline Center to corner distance of hexagonal clusters & $L=2 / \sqrt{3} R_{c}$ \\
\hline Scheduled users per BS & $K=3$ \\
\hline BS antennas & $M=3$ and 5 \\
\hline
\end{tabular}

of signal power introduced by increasing $R_{c}$ is negligible (as shown in Fig. 2). Therefore, increasing $R_{c}$ beyond the point that the interference power reaches the noise level does not yield any performance gain.

Similar to the previous case, the analytical results obtained from the expression in (12) are accurate and support and confirm the main conclusions of this paper.

\section{CONCLUding Remarks}

This paper investigates two distinct operating regimes of a downlink network MIMO system: a fully-loaded system with the same number of transmit and receive antennas within each cluster, and a system with an excess number of antennas at each BS. We show that increasing the cluster size causes two competing effects in a fully-loaded system. First, it reduces interference at the scheduled users. Second, it causes a penalty in terms of the signal power. Therefore, while increasing the cluster size improves the ergodic capacity of the cluster-edge users which are more prone to strong interference, the signal power penalty outweighs the gain in interference reduction at the users closer to the cluster center, thereby decreasing the center users' ergodic capacity. With $M>K$, however, increasing the cluster area is beneficial.

We further use tools from stochastic geometry to account for the random BS locations in characterizing the downlink ergodic capacity of network MIMO systems. In contrast to prior studies which rely on either simplified Wyner models 


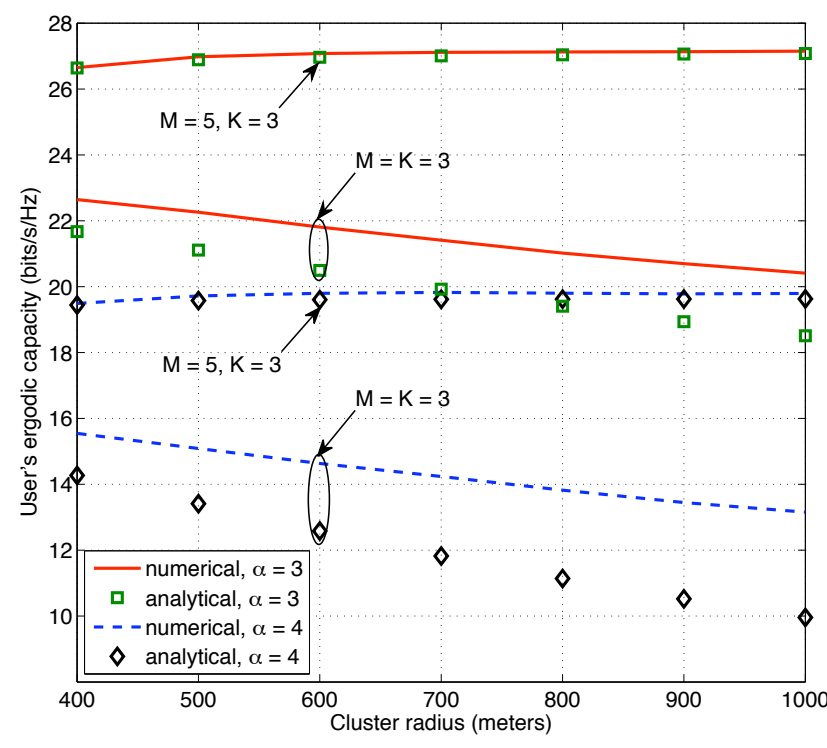

Fig. 2. Ergodic capacity of the user located at the cluster center as a function of cluster radius $R_{c}$ in a single-cluster network.

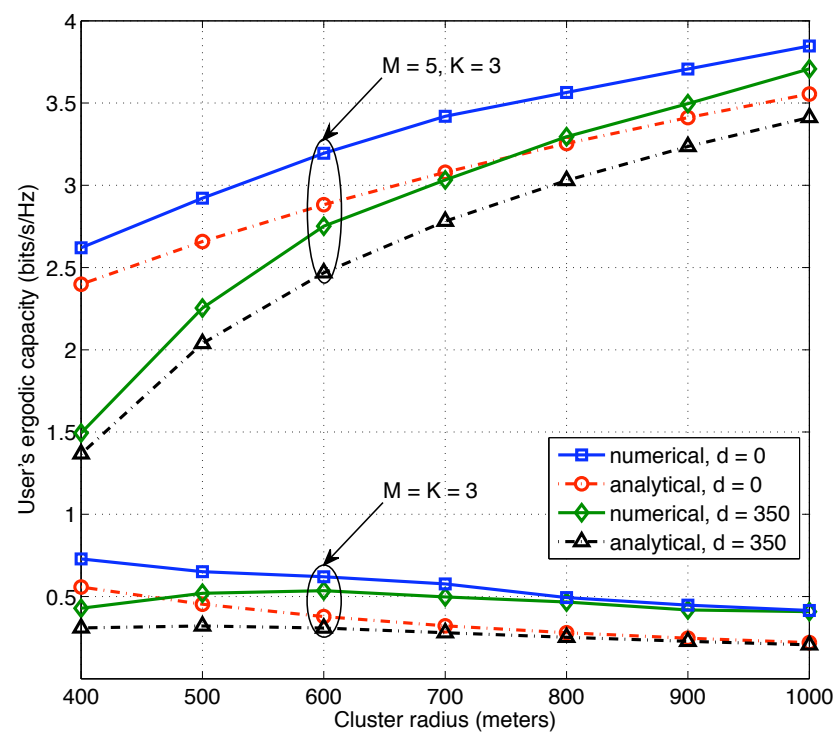

Fig. 3. User's ergodic capacity as a function of cluster radius $R_{c}$ for $d=0$ and $d=350$ meters in a multi-cluster network.

or extensive numerical simulations, this paper models the BS locations according to a homogeneous Poisson point process with a fixed density, and derives an efficiently computable ergodic capacity expression as a function of the distance between a user and the cluster center. This ergodic capacity expression is found to match the simulation results, and can be utilized to examine the performance of the network MIMO systems under various system parameters.

\section{REFERENCES}

[1] D. Gesbert, S. Hanly, H. Huang, S. S. Shitz, O. Simeone, and W. Yu, "Multi-cell MIMO cooperative networks: A new look at interference,"
IEEE J. Sel. Areas Commun., vol. 28, no. 9, pp. 1380-1408, Dec. 2010. [2] R. Tanbourgi, S. Singh, J. G. Andrews, and F. K. Jondral, "A tractable model for non-coherent joint transmission base station cooperation," 2014. [Online]. Available: http://arxiv.org/abs/1402.2707

[3] M. Haenggi, Stochastic geometry for wireless networks. Cambridge University Press, 2012.

[4] K. Huang and J. G. Andrews, "An analytical framework for multicell cooperation via stochastic geometry and large deviations," IEEE Trans. Inf. Theory, vol. 59, no. 4, pp. 2501-2516, 2013.

[5] Y. Lin and W. Yu, "Ergodic capacity analysis of downlink distributed antenna systems using stochastic geometry," Proc. IEEE Int. Conf. Commun. (ICC), pp. 3338-3343, June 2013.

[6] O. Simeone, O. Somekh, H. V. Poor, and S. Shamai, "Downlink multicell processing with limited-backhaul capacity," EURASIP J. Wireless Commun. and Netw., Feb. 2009.

[7] S. A. Ramprashad and G. Caire, "Cellular vs. network MIMO: A comparison including the channel state information overhead," Proc. IEEE Annu. Int. Symp. on Personal, Indoor, and Mobile Radio Commun. (PIMRC), pp. 878-884, Sep. 2009.

[8] N. Seifi, R. W. Heath, M. Coldrey, and T. Svensson, "Joint transmission mode and tilt adaptation in coordinated small-cell wireless networks," 2014. [Online]. Available: http://arxiv.org/abs/1407.1066

[9] K. Hosseini, W. Yu, and R. S. Adve, "Large-scale MIMO versus network MIMO for multicell interference mitigation," IEEE J. Sel. Topics in Signal Process., vol. 8, no. 5, pp. 930-941, Oct. 2014.

[10] V. Chandrasekhar, M. Kountouris, and J. G. Andrews, "Coverage in multi-antenna two-tier networks," IEEE Trans. Wireless Commun., vol. 8, no. 12, pp. 5314-5327, Oct. 2009.

[11] R. W. Heath, T. Wu, Y. H. Kwon, and A. C. K. Soong, "Multiuser MIMO in distributed antenna systems with out-of-cell interference," IEEE Trans. Signal Process., vol. 59, no. 10, pp. 4885-4899, Oct. 2011.

[12] T. Yoo and A. Goldsmith, "On the optimality of multiantenna broadcast scheduling using zero-forcing beamforming," IEEE J. Sel. Areas Commun., vol. 24, no. 3, pp. 528-541, Mar. 2006.

[13] K. A. Hamdi, "Capacity of MRC on correlated rician fading channels," IEEE Trans. Commun., vol. 56, no. 5, pp. 708-711, May 2008. 